\title{
Abnormal Cortical Plasticity in Youth with Autism Spectrum Disorder: A Transcranial Magnetic Stimulation Case-Control Pilot Study
}

\author{
Ernest V. Pedapati, MD,1,2 Donald L. Gilbert, MD, Craig A. Erickson, MD, \\ Paul S. Horn, PhD, ${ }^{2,3}$ Rebecca C. Shaffer, PsyD, Logan K. Wink, MD, \\ Cameron S. Laue, BS, and Steve W. Wu, MD²
}

\begin{abstract}
Objective: This case-control study investigated the use of a low-intensity repetitive transcranial magnetic stimulation (rTMS) protocol to measure motor cortex (M1) plasticity in youth with autism spectrum disorder (ASD) compared with typically developing children (TDC). We hypothesized that impairments in long-term potentiation-like properties represent a neurophysiological biomarker of abnormal cortical function in ASD.

Methods: We studied youth with ASD aged 11-18 years and matched controls (TDC). Intermittent theta burst stimulation (iTBS) was delivered to the dominant M1 at an intensity of $70 \%$ of resting motor threshold. Suprathreshold single-pulse TMS was performed to compare amplitudes of motor-evoked potentials (MEP) measured from surface electromyography electrodes on a target muscle before (20 pulses) and after (10 pulses/time point) iTBS at predefined timepoints (up to 30 minutes) to measure any potentiation effects. A linear mixed model was used to examine group differences in MEP amplitudes over time following iTBS. Results: Nine youth with ASD (mean age 15.6; 7 males; 6 right-hand dominant) and 9 TDC (mean age 14.5; 5 males; 9 righthand dominant) participated. All subjects tolerated the procedure well. Both groups had a mean increase in excitability after iTBS for 30 minutes; however, the time course of excitability changes differed $\left(F_{9,144}=2.05 ; p=0.038\right)$. Post-hoc testing identified a significant decrease in amplitude of the ASD group at 20 minutes following iTBS compared with the TDC after correcting for multiple comparisons.

Conclusion: In this study, we demonstrate early evidence for a potential physiological biomarker of cortical plasticity in youth with ASD using a rapid low-intensity rTMS protocol with a discriminate measure at 20 minutes following stimulation. The procedure was well tolerated by all 18 participants. Future work will include modification of the protocol to improve the ability to distinguish subtypes of ASD based on behavioral and cognitive testing.
\end{abstract}

\section{Introduction}

A UTISM SPECTRUM DISORDERS (ASD) consist of a range of pervasive developmental deficits marked specifically by delays in social, communication, and behavioral domains (APA 2013). The etiology of ASD is an area of intense investigation, and recent reports have suggested that abnormal cortical plasticity may play a key role in the pathophysiology of the disease (Walsh et al. 2008; Markram and Markram 2010; Oberman et al. 2014). The approach to viewing
ASD as a disorder of abnormal plasticity is supported by emerging data from translational models as well as preliminary human studies. Many putative genes associated with both idiopathic ASD (Gai et al. 2011) and monogenetic ASD syndrome (Kelleher III and Bear 2008) are involved in the development and function of synaptic connections (Wetmore and Garner 2010; Stephenson et al. 2011). In Shank2 $2^{-1-}$ knockout mice, an animal model of ASD, electrophysiological measurement of long-term potentiation (LTP) and long-term depression (LTD) in the hippocampus is severely impaired (Won et al. 2012).

Divisions of ${ }^{1}$ Neurology, ${ }^{2}$ Child and Adolescent Psychiatry, ${ }^{3}$ Biostatistics and Epidemiology, and ${ }^{4}$ Developmental and Behavioral Pediatrics, Cincinnati Children's Hospital Medical Center, Cincinnati, Ohio.

Funding: This study was supported by the Cincinnati Children's Hospital Medical Center Division of Neurology, Cincinnati Children's Hospital Research Foundation, and American Academy of Child and Adolescent Psychiatry Pilot Award.

(c) Ernest V. Pedapati et al. 2016; Published by Mary Ann Liebert, Inc. This Open Access article is distributed under the terms of the Creative Commons Attribution Noncommercial License (http://creativecommons.org/licenses/by-nc/4.0/) which permits any noncommercial use, distribution, and reproduction in any medium, provided the original author(s) and the source are credited. 
Recent human postmortem studies of individuals with low-functioning ASD and comorbid significant intellectual disability as well as fragile $X$ syndrome (FXS) have demonstrated immature dendritic morphology, including abnormally thin and tortuous dendritic spines (Belmonte and Bourgeron 2006).

From this perspective, ASD can be viewed as a developmental disorder, which emerges from a complicated interplay of network interaction among many brain regions and functional systems, rather than a discrete neuroanatomical or functional lesion (Belmonte et al. 2004). Thus, the presence of abnormal synapses and scaffolding poorly suited for activity-dependent changes and plasticitydependent maturation during critical periods contributes to the ASD phenotype (Walsh et al. 2008; LeBlanc and Fagiolini 2011). Despite the elegance of this "unified hypothesis," significant gaps must be addressed with empirical evidence. The present study uses transcranial magnetic stimulation (TMS), a form of noninvasive brain modulation, to address these gaps.

Theta burst stimulation (TBS) is a repetitive TMS (rTMS) protocol, which is capable of producing a robust, self-limited physiological plasticity effect in the human motor cortex (M1) and has been considered a surrogate method of inducing the cellular phenomena of LTP and LTD from the human cortex (Huang et al. 2005). In comparison to conventional rTMS, comparable physiological effects can be obtained with a TBS protocol that is shorter in duration and lower in intensity, favorable characteristics for use in sensitive populations such as children (Zafar et al. 2008; Hong et al. 2015). When applied to M1, intermittent TBS (iTBS) can result in facilitation (LTP-like effect) of motor evoked potentials (MEP) peak-to-peak amplitudes for 10-90 minutes following stimulation. In contrast, continuous TBS may lead to MEP amplitude suppression (LTD-like effect) following stimulation (Huang et al. 2005; Wu et al. 2012).

Previously, using a 300 pulse, $30-\mathrm{Hz}$ intermittent TBS protocol (Pedapati et al. 2015), we have demonstrated a robust facilitation of M1 cortical excitability in a cohort of healthy children. In this pilot study, we were interested in using a case-control design to compare the effects of iTBS on cortical excitability between typically developing children (TDC) and children with ASD. The overall goal of this work was to investigate a potential safe and robust biomarker to grossly quantify the final output of M1 and potentially use as a measure to modulate these networks and track treatment response. We hypothesized based on previous studies (Oberman et al. 2010, 2014) that ASD subjects would demonstrate excessive facilitation compared with a cohort of age-matched TDC.

\section{Methods}

Parent(s) of pediatric patients gave written informed consent and child participants gave written informed assent for the study, which was approved by the Cincinnati Children's Hospital Medical Center Institutional Review Board. Participants were reimbursed for time and travel.

\section{Study subjects}

Children with and without ASD between the ages 8 and 18 years were recruited through an outpatient ASD clinical treatment population. Diagnosis of ASD was confirmed by previous Autism Diagnostic Observation Schedule, Second Edition (ADOS-2; Lord et al. 2012), and intelligence testing (Roid 2003; Elliott 2007) was additionally performed by an experienced clinician. Potential subjects with an IQ less than 80 were excluded from the study. For this pilot study, ASD subjects with comorbid psychiatric conditions and medications were permitted in the ASD group; however, subjects had to be on stable doses of medications for at least 4 weeks. Subjects on medications known to affect M1 plasticity were excluded. The TDC comparison group was drawn from a sample previously published (Pedapati et al. 2015) and was free of neurologic, psychiatric, and serious medical illnesses; was not taking any neuropsychiatric medications; and did not have any contraindications to TMS including history of epilepsy (Rossi et al. 2011). Nine TDC subjects were selected to closely match the ASD subjects based on age and gender. Handedness was either determined through Physical And Neurological Examination for Soft Signs (PANESS) (Denckla 1985) or the Edinburgh Handedness Inventory (Oldfield 1971).

\section{Procedure}

Measurement of resting motor threshold. Surface electromyography (EMG) was placed over the first dorsal interosseous (FDI) muscle of the dominant hand. Participants were seated comfortably with pillows to support both arms fully. Full muscle relaxation was monitored by EMG and visual inspection of muscle tone. Magstim Super Rapid ${ }^{2}$ Plus ${ }^{1}$ stimulator (Magstim Co., Wales, United Kingdom) connected to 70-mm figure-8 coils was used to perform rTMS and a Magstim 200 stimulator was used to perform single-pulse (spTMS) stimulation to obtain MEPs, as previously described (Pedapati et al. 2015). The figure- 8 coil (handle pointing posteriorly at $45^{\circ}$ ) was placed tangentially to the scalp over the dominant primary $\mathrm{M} 1$ at the optimal site for obtaining maximal peak-to-peak amplitude of MEPs from the dominant FDI. A wax pencil was used to mark this "hot spot" for consistent placement of the coil during spTMS and rTMS. We measured resting motor threshold (RMT) for each Magstim stimulator separately as the minimal intensity needed to induce MEPs in at least three of six consecutive trials (Mills and Nithi 1997). Test pulse intensity was $\sim 120 \% *$ RMT. Baseline MEP amplitude was measured by 20 TMS test pulses separated by 6 seconds $( \pm 5 \%)$.

Intermittent TBS. Based on previous methods, Magstim Super Rapid ${ }^{2}$ Plus ${ }^{1}$ connected to a figure- 8 coil was used to perform iTBS (Pedapati et al. 2015). We recently discussed the development of this protocol, including stimulation duration and intensity, as an optimization of conventional iTBS for pediatric subjects (Huang et al. 2005; Pedapati et al. 2015). All iTBS sessions were performed in the afternoon. Application of iTBS was applied to the designated hot spot. A modified iTBS protocol consisted of bursts of 3 pulses at $30 \mathrm{~Hz}$ repeating every $200 \mathrm{~ms}$ for 2 seconds (1 train) with trains repeating every 10 seconds apart for a total of 300 pulses. Stimulation intensity of iTBS was $70 \%$ of RMT, rather than based on active motor threshold, as phasic and tonic finger movements can influence the outcome of TBS (Gentner et al. 2008).

\section{Safety and tolerability assessment}

Assessment for adverse events were conducted before and after TMS using a 16-question review of systems, which included headache, scalp pain, arm/hand pain, other pain(s), numbness/ tingling, other sensation(s), weakness, loss of dexterity, vision/ hearing change(s), ear ringing, nausea/vomiting, appetite loss, rash, skin change(s), or any other symptom(s) on a scale of 0-5 (none, minimal, mild, moderate, marked, severe) (Hong et al. 2015).

\section{Statistical analyses}

Descriptive statistics were applied to demographic and baseline TMS measures. The participants' age between the TDC and ASD 
groups were compared using the Mann-Whitney $U$-test. The Fisher exact test was used to compare proportion of gender between the groups. All analyses were performed in SAS $^{\circledR}$ (SAS Institute, Inc., Cary, NC) with a two-tailed $p<0.05$ considered significant. Linear mixed model (LMM): We conducted a repeated-measures LMM using the PROC-MIXED procedure (SAS version 9.3; SAS Institute) with a compound symmetry covariance structure (the best of four structures examined) to examine group differences in MEP amplitudes following iTBS.

The LMM was used to model all raw MEP amplitudes (mV) of each subject (the random effect) and incorporate baseline (pre-iTBS) amplitude as a covariate. Time was used as the repeated factor to analyze response from 1 to 30 minutes (TIME; 10 levels). Thus, the fixed effects were Group, Time, Group $\times$ Time interaction, and baseline. After post-hoc comparison of each time-point against baseline, the resultant $p$-value was corrected for multiple comparisons (Tukey-Kramer). We anticipated as our primary hypothesis that the Group $\times$ Time interaction would differ between the groups. Area under the curve $(A U C)$ : For each subject, the mean post-iTBS amplitudes were standardized to proportional change from baseline (i.e., normalized as a proportion of mean baseline amplitude and then adjusted by subtracting one). Total AUC was defined as the sum of individual calculated trapezoidal areas between consecutive time points from 0 (post-iTBS) to 30 minutes. Facilitation as defined as a positive AUC value and suppression as a negative AUC value. Pearson product-moment correlation was used to identify significant relationships between variables of interest.

\section{Results}

Of a total of 11 subjects recruited, 9 youth with ASD (7 males, 6 right-hand dominant) completed the study and were matched with 9 age-matched controls ( 5 males, 9 right-hand dominant) for the final analysis. Two subjects taking memantine were excluded from analysis, given potential modulatory effects on iTBS (Huang et al. 2007). There was no significant difference in age $\left(\mathrm{T}_{16}=1.06, p=0.31\right)$. No moderate or severe adverse events were identified by structured diagnostic interviews and no seizures occurred. One subject reported a mild headache (rated: minimal) following iTBS, which spontaneously resolved. Summary demographics and clinical measures are listed in Table 1. Individual subject demographics, comorbid psychiatric conditions, and medications are listed in Table 2.

The majority of TDC (6/9) and ASD (6/9) subjects demonstrated a positive AUC estimate following iTBS over 30 minutes (see AUC, Table 2). A robust interpretation of an AUC is limited, as it

Table 1. Subject Characteristics

\begin{tabular}{lccc}
\hline $\begin{array}{l}\text { Measurements, } \\
\text { mean }(S D)\end{array}$ & ASD $(\mathrm{n}=9)$ & $T D C(\mathrm{n}=9)$ & Statistic, $\mathrm{p}$ \\
\hline Age in years & $15.6(1.8)$ & $14.5(2.2)$ & $U=29.5 ; p=0.35$ \\
Range & $13-18$ & $11-18$ & \\
Gender & 7 males & 5 males & $p=0.62^{\mathrm{a}}$ \\
IQ & $106.2(16.2)$ & & \\
ADOS-SA & $8.9(2.8)$ & & \\
ADOS-RRB & $3.3(2.2)$ & & \\
ADOS-total & $12.2(3.3)$ & & \\
\hline
\end{tabular}

${ }^{a}$ Fisher's exact test.

ADOS, autism diagnostic observation schedule; ASD, autism spectrum disorder group; IQ, intelligence quotient; RRB, restrictive and repetitive behavior score; SA, social affect score; SD, standard deviation; TDC, typically developing children.
Table 2. Sample Characteristics and Area UNDER THE ITBS Response CURVE

\begin{tabular}{|c|c|c|c|c|}
\hline \multicolumn{5}{|c|}{ ASD subjects } \\
\hline Age & Sex & $A U C$ & Comorbid Dx & Medications \\
\hline 13 & M & 4.1 & ODD & Risperidone \\
\hline 13 & $\mathrm{~F}$ & 15.2 & None & \\
\hline 15 & $\mathrm{~F}$ & 19.3 & Anxiety, ADHD & $\begin{array}{l}\text { D-amphetamine, } \\
\text { duloxetine }\end{array}$ \\
\hline 15 & M & -42.2 & Anxiety, ADHD & $\begin{array}{l}\text { Citalopram, MPH, } \\
\text { clonidine }\end{array}$ \\
\hline 15 & M & -9.5 & OCD & \\
\hline 17 & M & -1.6 & Anxiety & \\
\hline 17 & M & 36.1 & OCD & \\
\hline 17 & M & 31.3 & Anxiety & \\
\hline 18 & M & 13.8 & None & Citalopram \\
\hline
\end{tabular}

TDC subjects

\begin{tabular}{llr}
\hline 11 & M & 2.6 \\
13 & $\mathrm{M}$ & 32.5 \\
13 & $\mathrm{~F}$ & -31.5 \\
14 & $\mathrm{M}$ & -27.3 \\
14 & $\mathrm{M}$ & 11.2 \\
15 & $\mathrm{~F}$ & 11.9 \\
16 & $\mathrm{M}$ & 90.4 \\
17 & $\mathrm{~F}$ & 2.3 \\
18 & $\mathrm{~F}$ & -8.4 \\
\hline
\end{tabular}

ADHD, attention-deficit/hyperactivity disorder; ASD, autism spectrum disorder; AUC, area under the response curve; Dx, diagnosis; iTBS, intermittent theta burst stimulation; $\mathrm{MPH}$, methylphenidate; $\mathrm{OCD}$, obsessive compulsive disorder; ODD, oppositional defiant disorder; TDC, typically developing children.

does not account for time course, that is, facilitation early in the time course can be offset by later suppression. As expected, there was no significant group difference between baseline raw MEP amplitudes, RMT, or iTBS stimulus intensity (Table 3). At baseline, the LMM analysis identified a significant intersubject differences in raw MEP amplitudes $\left(F_{1,15}=0.44 ; p=0.52\right)$. Therefore, this was included as a covariate in the model. A significant Time $\times$ Group interaction was identified $\left(F_{9,144}=2.05 ; p=0.038\right)$, while neither Group $\left(F_{1,15}=0.44 ; p=0.52\right)$ nor TIME $\left(F_{9,144}=0.75\right.$; $p=0.67$ ) alone were statistically significant. Post-hoc testing identified a significant decrease in amplitude of the ASD group at 20 minutes following iTBS compared with the TDC after correcting for multiple comparisons (Tukey-Kramer; $t=-2.37 ; p=0.02$ ). Full results of the LMM findings, including post-hoc comparisons, are listed in Table 3. Adding age as a covariate did not have a significant effect in either analysis (data not shown). The mean modeled MEP amplitude estimates for each time point by group are illustrated in Figure 1. No significant difference of mean AUC between the groups was identified $(t=0.13 ; p=0.89)$. No significant relationship was found between IQ and AUC $(r=0.46$, $p=0.21)$, ADOS social affect total $(r=0.38, p=0.31)$, ADOS restrictive and repetitive behavior total $(r=-0.46, p=0.22)$, and ADOS total score $(r=0.02, p=0.95)$.

\section{Discussion}

Intermittent TBS, a TMS technique to date primarily used in adults, was used in this study to quantify LTP-like excitability in the M1 in children with ASD. We found evidence of a difference 
TABle 3. TMS Characteristics AND LMM Results

\begin{tabular}{|c|c|c|c|c|c|c|c|c|c|c|}
\hline \multicolumn{11}{|c|}{ TMS characteristics } \\
\hline \multicolumn{4}{|l|}{ Measurement } & \multicolumn{2}{|l|}{$A S D(\mathrm{n}=9)$} & \multicolumn{2}{|c|}{$T D C(\mathrm{n}=9)$} & & \multicolumn{2}{|c|}{ Statistic, $\mathrm{p}$} \\
\hline \multirow{3}{*}{\multicolumn{4}{|c|}{$\begin{array}{l}\text { Baseline MEP, mV } \\
\text { RMT (Magstim Super Rapid }{ }^{2} \text { Plus }^{1} \text { ) } \\
\text { iTBS intensity }\end{array}$}} & \multirow{3}{*}{\multicolumn{2}{|c|}{$\begin{array}{r}1.9(1.12) \\
64.6(15.8) \\
45.3(11.1)\end{array}$}} & \multirow{3}{*}{\multicolumn{2}{|c|}{$\begin{aligned} & 2.8(1.7) \\
63.2 & (10.8) \\
44.2 & (7.6)\end{aligned}$}} & & \multirow{3}{*}{\multicolumn{2}{|c|}{$\begin{array}{r}t_{16}=-1.29 ; p=0.22 \\
t_{16}=0.21 ; p=0.84 \\
t_{16}=0.25 ; p=0.81\end{array}$}} \\
\hline & & & & & & & & & & \\
\hline & & & & & & & & & & \\
\hline \multicolumn{11}{|c|}{$L M M$ results and post-hoc analysis } \\
\hline \multicolumn{3}{|l|}{ Fixed effect } & \multicolumn{2}{|c|}{$D F$} & \multicolumn{4}{|c|}{ F-value } & & $\mathrm{p}$ \\
\hline \multirow{4}{*}{\multicolumn{3}{|c|}{$\begin{array}{l}\text { Baseline (subject) } \\
\text { Group } \\
\text { Time } \\
\text { Time } \times \text { group }\end{array}$}} & \multicolumn{2}{|c|}{1,15} & \multicolumn{4}{|c|}{22.32} & & $<0.001^{\mathrm{a}}$ \\
\hline & & & \multicolumn{2}{|c|}{1,15} & \multicolumn{4}{|c|}{0.44} & & 0.52 \\
\hline & & & \multirow{2}{*}{\multicolumn{2}{|c|}{$\begin{array}{l}9,144 \\
9,144\end{array}$}} & \multicolumn{4}{|c|}{0.75} & & 0.67 \\
\hline & & & & & & & 05 & & & $0.04^{\mathrm{a}}$ \\
\hline \multicolumn{11}{|c|}{ Post-hoc comparisons for Time $\times$ Group } \\
\hline $\begin{array}{l}\text { Timepoint } \\
\text { df }\end{array}$ & 1 & \multicolumn{8}{|c|}{$1,68.85$} & 30 \\
\hline$F$-value & 1.24 & 0.95 & 0.57 & 0.54 & 0.14 & 1.36 & 1.22 & 0.25 & 5.6 & 1.71 \\
\hline $\begin{array}{l}p \\
\text { Adjusted } p\end{array}$ & 0.27 & 0.33 & 0.45 & 0.46 & 0.71 & 0.25 & 0.27 & 0.62 & $\begin{array}{l}0.02^{\mathrm{a}} \\
0.019^{\mathrm{b}}\end{array}$ & 0.20 \\
\hline
\end{tabular}

Timepoints are in minutes after TBS.

${ }^{a}$ Statistical significance.

${ }^{\mathrm{b}} p$ After adjustment for multiple comparisons (Tukey-Kramer).

$\mathrm{t}_{68.85}=-2.37$ (Kenward-Roger approximation for the degrees of freedom).

ASD, autism spectrum disorder; LMM, linear mixed model; MEP, motor-evoked potential amplitude; RMT, resting motor threshold as percentage of maximal stimulator output; TDC, typically developing children; TMS, transcranial magnetic stimulation.

during the post-iTBS time course of M1 excitability between youth with ASD and their typically developing peers. Recent studies have reported differences in TMS metrics associated with cortical plasticity in adults with ASD (Oberman et al. 2012; Jung et al. 2013), but this is the first study looking at the effects of iTBS in ASD during the developmental period. The interpretation of the relationship between neuroplasticity and disease pathophysiology with these intriguing findings requires additional study.

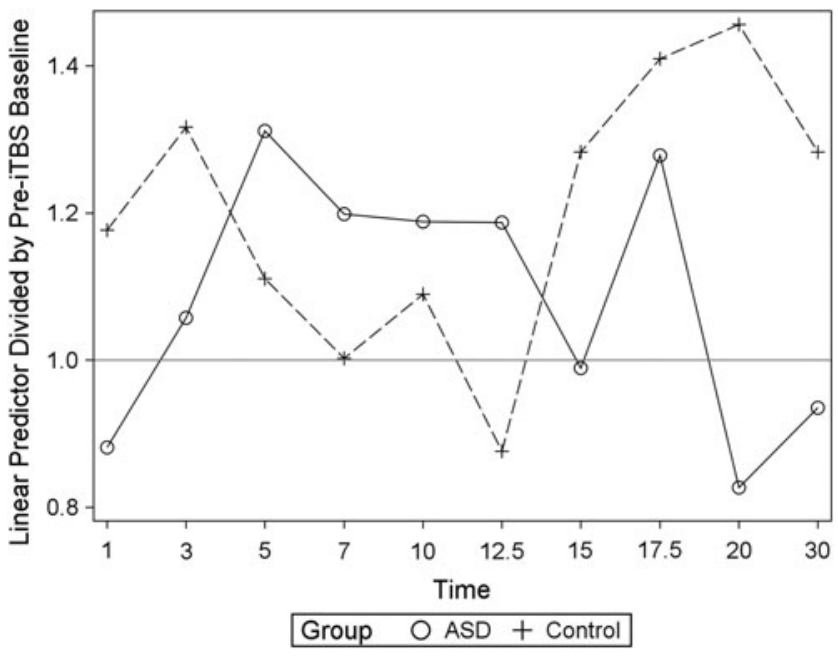

FIG. 1. Linear mixed model estimates of post-iTBS motor-evoked potential amplitude over baseline for each timepoint (minutes) by group. Post-hoc comparison indicated a significant difference at 20 minutes, with ASD demonstrating significantly less facilitation than TDC (Control). ASD, autism spectrum disorder group; Control, typically developing children; iTBS, intermittent theta burst stimulation.
There has been increasing interest in the study of motor system abnormalities in ASD. These abnormalities have been frequently identified in persons with ASD across their lifespan and could be predictive of the disorder (Leary and Hill 1996; Fabbri-Destro et al. 2013). For example, compared to controls, infants later diagnosed with ASD have movement abnormalities involving reflexes, gait sequencing, hand movements, and crawling before observed deficits in social and language difficulties (Teitelbaum et al. 1998). In a standardized test of motor proficiency, motor skills were severely impaired among ASD-affected children compared with unaffected siblings. These findings correlated with autism severity and IQ (Hilton et al. 2012). These results have been further supported by a meta-analysis of 83 studies examining motor function in ASD, which demonstrated a large group effect size, suggesting that motor deficits are a cardinal feature of ASD (Fournier et al. 2010).

Based on the hypothesis that the motor system develops differently in ASD, we used repetitive TMS to probe the M1 using a protocol (iTBS) previously shown to produced LTP-like changes in cortical excitability in children (Pedapati et al. 2015). Although the majority of TDC (6/9) and ASD (6/9) subjects demonstrated facilitation of MEP amplitudes following iTBS over 30 minutes (see AUC, Table 2), at 20 minutes, ASD subjects' MEP amplitudes were significantly depressed compared with the TDC cohort. Jung et al. (2013) also found a diminished LTP-like plasticity in high-functioning young adults with ASD in response to paired associative stimulation (PAS). In a study examining the after effects of iTBS in adults with ASD, Oberman et al. (2012) also found an abnormal time course, but not diminished amplitudes, as facilitation was longer lasting in ASD than matched controls.

Although different physiological mechanisms could be involved in PAS versus iTBS, both are thought to involve changes in neurotransmission within the cortex to induce facilitatory neuroplasticity in healthy subjects (Huang et al. 2005; Player et al. 2012). The current findings suggest that under certain parameters, iTBS may 
discriminate between ASD and TDC populations. However, with the current data set, we can only speculate that these changes reflect abnormalities in cortical excitability in ASD. To further investigate if these changes reflect differences in absolute differences at each time point or differences in latency (i.e., a shift of the response curve), future studies may need to extend recording time until signal returns to baseline. Other TMS studies using single- and paired-pulse measures, including input-output curves, intracortical inhibition and facilitation, and motor threshold, have found no difference between ASD and control samples on the whole (Théoret et al. 2005; Enticott et al. 2013). Of interest, however, is that differences may be present between certain subgroups such as those with early language delay (Théoret et al. 2005; Enticott et al. 2013) or younger age (Enticott et al. 2010).

Wide interindividual variability in TMS techniques to probe plasticity, including TBS (Hamada et al. 2012; Hinder et al. 2014) and PAS (Damji et al. 2015), has been previously reported in healthy controls. At times the phenomenon of interest, a post-TBS change in excitability, is absent in healthy subjects (Hamada et al. 2012; Player et al. 2012; Vernet et al. 2014; Pedapatiet al. 2015). The physiological mechanism of iTBS are theorized to result from an increased excitability in the circuitry of the M1, which leads to increased facilitation of trans-synaptic activation of corticospinal neurons and may be influenced by intrinsic individual variation (Huang et al. 2007; Di Lazzaro et al. 2008). Response variability in ASD could be further complicated by the inherent heterogeneity, including differences in underlying physiology, cognitive phenotypes, and medical and psychiatric comorbidities. The use of other techniques, such as electroencephalography or neuroimaging, may complement further, clarifying a range of abnormal neuroplasticity and behavioral findings in autism (Belmonte et al. 2004; Casanova et al. 2012; Jung et al. 2013; Vernet et al. 2013; Wang et al. 2013). For example, imaging techniques, such as MR Spectroscopy for GABA (Yang et al. 2014), can be productively combined with TMS plasticity studies, especially as GABA abnormalities may play a key role in the pathophysiology of ASD (Coghlan et al. 2012).

Of interest, adult TBS studies have shown reasonable reproducibility between sessions of the same individuals, which suggests the technique better suited for within subject experimental designs (Hinder et al. 2014). In an analysis of post-iTBS variability between two separate visits in 30 healthy adults, Hinder et al. found that $41.4 \%$ of the total variance in the iTBS response was attributed to interindividual differences with only $12.6 \%$ of the total variance due to intraindividual variability (Hinder et al. 2014). This raises the possibility that TBS may be useful in examining intraindividual changes before and after intervention, including medication or therapy. However, these findings may not easily translate into clinical populations and children, in which additional physiological variability contributing to TMS metrics have not been fully characterized. If sufficient progress is made in the optimization of these techniques, including the identification of key factors influencing variability, TMS measures in general, including TBS, may have utility as a functional assay to predict or measure pharmacological response (Frazier et al. 2011; Ziemann et al. 2015).

Further refining of these techniques and sample selection will be necessary to establish iTBS-evoked changes in excitability as a biomarker in ASD. The current findings will need to be replicated in a larger sample size, ideally across multiple laboratories using a similar case-control design with medication-naive patients. The adoption of consistent techniques across laboratories in terms of stimulation parameters could potentially accelerate this process. It could be prudent for some studies to narrow the subject sample to monogenetic ASD syndrome, such as FXS. Monogenetic disorders are particularly advantageous for clinical investigation as animal and in vitro models of the specific genetic deficits can be replicated and invasively studied and treated (Spooren et al. 2012).

It is also crucial that these TMS measures correlate with behavioral phenotypes, such that standardized ASD measures including symptom severity scores, neurobehavioral markers (i.e., eye tracking or behavioral tasks), or treatment responses will be identified. Although we did not find evidence of a relationship between iTBS response and behavioral measure, the current sample was not sufficiently powered to detect a difference. If such relationships are identified, this may assist further in clarifying interindividual response variability to TMS. In larger samples, strategically TMSderived measures may elucidate a variety of pathophysiological processes, which underlie the generation of ASD symptoms and delineate subtypes (De Rubeis and Buxbaum 2015). Finally, due, in part, to safety concerns, there are limited data in healthy children, and certain populations (i.e., epilepsy) may ultimately be excluded from future studies. As safety data accumulate, the feasibility of multiple centers to acquire normative pediatric data increases (Wu et al. 2012; Honget al. 2015; Krishnan et al. 2015).

In the current protocol, repetitive TMS using iTBS was used primarily as a cortical probe, rather than as an interventional technique. Although the majority of studies examining the use of TMS as an interventional tool are small and uncontrolled (Oberman et al. 2013), recently a double-blind sham-controlled trial of deep rTMS in adults with ASD demonstrated a modest improvement in self-reported measures of autism specific symptoms (Enticott et al. 2014). In rats, iTBS has been associated with improved learning performance and led to increases in cortical protein expression (c-Fos, GAD65, and parvalbumin) associated with synaptic plasticity (Funke and, Benali 2011). More recently in human subjects, iTBS delivered to the M1 led to enhancement of functional connectivity between M1 and the ipsilateral dorsal premotor cortex, in addition to previously characterized changes in MEP amplitudes (Nettekoven et al. 2014). Taken together, there is sufficient scientific rationale to suggest that future efforts should consider the potential neuromodulatory effects of iTBS, perhaps by pairing stimulation with a behavioral task relevant in ASD (eye tracking or cognitive testing) or a serum biomarker (Erickson et al. 2011).

\section{Limitations}

Several important limitations apply to this study and limit generalizability until replication. The results of this study are vulnerable to a type II error, given the small sample size. The ASD sample was heterogeneous with regard to comorbid psychiatric conditions and some were taking psychiatric medications, which can affect TMS response. Some comorbid diagnoses, such as ADHD, may have unique TMS profiles, which may confound the current results. In addition, differences in cortical excitability and inhibition between right- and left-handed individuals have been reported (Reid and Serrien 2012; Daligadu et al. 2013; Davidson and Tremblay 2013). Although we aimed to broaden recruitment by including left-handed individuals, this may have introduced additional variability. Due to limited control data, TDC subjects were not matched 1:1 for gender and age, but matched on average.

Although no ASD subjects had intellectual disability in this study, IQ matching was not performed. A prospective case-control series with strict matching may yield different results. As no sham 
condition was used, this may also be a nonspecific TMS or time effect. In addition, repeated sessions could be used to evaluate any intraindividual variability, for example, related to time of day, stress, fatigue, or hormonal fluctuations in females.

\section{Conclusions and Clinical Significance}

In this report, we present the results of a pilot study comparing iTBSinduced M1 excitability between a cohort of high-functioning ASD youth and age- and gender-matched typically developing youth. Although both groups, on average, demonstrated facilitation of MEP amplitudes, at 20 minutes, MEP amplitudes in the ASD group were diminished compared with the TDC group. Along with other recent reports of TMS use in ASD (Obermanet al. 2012; Enticott et al. 2013; Jung et al. 2013; Oberman et al. 2014), TBS may represent a pathway for investigating suspected abnormalities in cortical excitability in ASD.

\section{Disclosures}

All contributing authors have read and approved the submission of this manuscript to the journal. Additionally, all authors report no direct conflicts with the content of this report. Dr. Pedapati receives research support from the Cincinnati Children's Hospital Research Foundation and the American Academy Child and Adolescent Psychiatry. Dr. Gilbert has received honoraria from the Tourette Syndrome Association/Centers for Disease Control and Prevention and the American Academy of Pediatrics, serves on the medical advisory board for the Tourette Syndrome Association, has received book royalties from Elsevier. Dr. Gilbert has received research support (for Tourette Syndrome, ADHD) from the NIH (NIMH R01 MH092520, NIMH R01 MH081854, NIMH R01 104651, NINDS R01 NS085023), from Ecopipam Pharmaceuticals (clinical trial, Tourette Syndrome). Dr. Erickson is a consultant to and holds equity in Confluence Pharmaceuticals and is a consultant to Alcobra Pharmaceuticals. Dr. Erickson is a past consultant to the Roche Group and Novartis. Dr. Erickson holds nonrelated IP held by CCHMC and Indiana University. Dr. Erickson receives research grant support from the John Merck Fund, CCHMC, Autism Speaks, the National Fragile X Foundation, The Roche Group, Neuren Pharmaceuticals, Synapdx, and Riovant Sciences Ltd. Dr. Horn receives research support from the NIH/NINDS and the John Merck Fund. Dr. Shaffer has no disclosures to report. Dr. Wink's current research is supported by the Simons Research Foundation, the John Merck Fund, United Stated Department of Defense, Autism Speaks, SynapDx, and Riovant Sciences Ltd. Dr. Wink has served as a past consultant for Otsuka. Cameron Laue has no disclosures to report. Dr. Wu receives research support from NIH (R01MH078160) and from Ecopipam Pharmaceuticals (clinical trial, Tourette Syndrome).

\section{References}

American Psychiatric Association: Diagnostic and Statistical Manual of Mental Disorders, 5th ed. (DSM-5). Washington, DC: American Psychiatric Publishing; 2013.

Belmonte MK, Allen G, Beckel-Mitchener A, Boulanger LM, Carper RA, Webb SJ: Autism and abnormal development of brain connectivity. J Neurosci 24:9228-9231, 2004.

Belmonte MK, Bourgeron T: Fragile X syndrome and autism at the intersection of genetic and neural networks. Nat Neurosci 9: 1221-1225, 2006.

Casanova MF, Baruth JM, El-Baz A, Tasman A, Sears L, Sokhadze E: Repetitive transcranial magnetic stimulation (rTMS) modulates event-related potential (ERP) indices of attention in autism. Transl Neurosci 3:170-180, 2012.
Coghlan S, Horder J, Inkster B, Mendez MA, Murphy DG, Nutt DJ: GABA system dysfunction in autism and related disorders: From synapse to symptoms. Neurosci Biobehav Rev 36:2044-2055, 2012.

Daligadu J, Murphy B, Brown J, Rae B, Yielder P: TMS stimulusresponse asymmetry in left- and right-handed individuals. Exp Brain Res 224:411-416, 2013.

Damji O, Keess J, Kirton A. Evaluating developmental motor plasticity with paired afferent stimulation. Dev Med Child Neurol 57: 548-555, 2015.

Davidson T, Tremblay F: Hemispheric differences in corticospinal excitability and in transcallosal inhibition in relation to degree of handedness. PLoS One 8:e70286, 2013.

Denckla MB: Revised neurological examination for subtle signs (1985). Psychopharmacol Bull 21:773-800, 1985.

De Rubeis $\mathrm{S}$ and Buxbaum JD. Genetics and genomics of autism spectrum disorder: Embracing complexity. Hum Mol Genet 24:R24-31, 2015.

Di Lazzaro V, Pilato F, Dileone M, Profice P, Oliviero A, Mazzone P, Insola A, Ranieri F, Meglio M, Tonali P: The physiological basis of the effects of intermittent theta burst stimulation of the human motor cortex. J Physiol 586:3871-3879, 2008.

Elliott CD: Differential Ability Scales-Second edition (DAS-II). San Antonio (Texas), Harcourt Assessment, 2007.

Enticott PG, Fitzgibbon BM, Kennedy HA, Arnold SL, Elliot D, Peachey A, Zangen A, Fitzgerald PB: A double-blind, randomized trial of deep repetitive transcranial magnetic stimulation (rTMS) for autism spectrum disorder. Brain Stimul 7:206-211, 2014.

Enticott PG, Kennedy HA, Rinehart NJ, Tonge BJ, Bradshaw JL, Fitzgerald PB: GABAergic activity in autism spectrum disorders: An investigation of cortical inhibition via transcranial magnetic stimulation. Neuropharmacol 68:202-209, 2013.

Enticott PG, Rinehart NJ, Tonge BJ, Bradshaw JL, Fitzgerald PB: A preliminary transcranial magnetic stimulation study of cortical inhibition and excitability in high-functioning autism and Asperger disorder. Dev Med Child Neurol 52:e179-183, 2010.

Erickson CA, Weng N, Weiler IJ, Greenough WT, Stigler KA, Wink LK, McDougle CJ: Open-label riluzole in fragile $\mathrm{X}$ syndrome. Brain Res 1380:264-270, 2011.

Fabbri-Destro M, Gizzonio V, Avanzini P: Autism, motor dysfunctions and mirror mechanism. Clin Neuropsychiatry 10:177-187, 2013.

Fournier K, Hass C, Naik S, Lodha N, Cauraugh J: Motor coordination in autism spectrum disorders: A synthesis and meta-analysis. J Autism Dev Disord 40:1227-1240, 2010.

Frazier TW, Shattuck PT, Narendorf SC, Cooper BP, Wagner M, Spitznagel EL: Prevalence and correlates of psychotropic medication use in adolescents with an autism spectrum disorder with and without caregiver-reported attention-deficit/hyperactivity disorder. J Child Adolesc Psychopharmacol 21:571-579, 2011.

Funke K, Benali A: Modulation of cortical inhibition by rTMS-findings obtained from animal models. J Physiol 589:4423-4435, 2011.

Gai X, Xie H, Perin J, Takahashi N, Murphy K, Wenocur A, D'arcy M, O'Hara R, Goldmuntz E, Grice D: Rare structural variation of synapse and neurotransmission genes in autism. Mol Psychiatry 17:402-411, 2011.

Gentner R, Wankerl K, Reinsberger C, Zeller D, Classen J: Depression of human corticospinal excitability induced by magnetic thetaburst stimulation: Evidence of rapid polarity-reversing metaplasticity. Cereb Cortex 18:2046-2053, 2008.

Hamada M, Murase N, Hasan A, Balaratnam M, Rothwell JC: The role of interneuron networks in driving human motor cortical plasticity. Cereb Cortex 23: 1593-1605, 2013.

Hilton CL, Zhang Y, Whilte MR, Klohr CL, Constantino J: Motor impairment in sibling pairs concordant and discordant for autism spectrum disorders. Autism 16:430-441, 2012. 
Hinder MR, Goss EL, Fujiyama H, Canty AJ, Garry MI, Rodger J, Summers JJ: Inter-and intra-individual variability following intermittent theta burst stimulation: Implications for rehabilitation and recovery. Brain stimulation 7:365-371, 2014.

Hong YH, Wu SW, Pedapati EV, Horn PS, Huddleston DA, Laue CS, Gilbert DL: Safety and tolerability of theta burst stimulation versus single and paired pulse transcranial magnetic stimulation: A comparative study of 165 pediatric subjects. Front Hum Neurosci 9:29, 2015.

Huang YZ, Chen RS, Rothwell JC, Wen HY: The after-effect of human theta burst stimulation is NMDA receptor dependent. Clin Neurophysiol 118:1028-1032, 2007.

Huang YZ, Edwards MJ, Rounis E, Bhatia KP, Rothwell JC: Theta burst stimulation of the human motor cortex. Neuron. 45:201-206, 2005.

Jung NH, Janzarik WG, Delvendahl I, Munchau A, Biscaldi M, Mainberger F, Baumer T, Rauh R, Mall V: Impaired induction of long-term potentiation-like plasticity in patients with high-functioning autism and Asperger syndrome. Dev Med Child Neurol. 55:83-89, 2013.

Kelleher III RJ, Bear MF: The autistic neuron: Troubled translation? Cell 135:401-406, 2008.

Krishnan C, Santos L, Peterson MD, Ehinger M: Safety of noninvasive brain stimulation in children and adolescents. Brain stimulation 8:76-87, 2015.

Leary MR, Hill DA: Moving on: Autism and movement disturbance. Ment Retard 34:39-53, 1996.

LeBlanc JJ, Fagiolini M: Autism: A "critical period" disorder? Neural Plast 2011: Article ID 921680, 2011.

Lord C, Rutter M, DiLavore PC, Risi S, Gotham K and Bishop S: Autism Diagnostic Observation Schedule, Second Edition: ADOS-2. Los Angeles, CA: Western Psychological Services; 2012.

Markram K, Markram H: The intense world theory—a unifying theory of the neurobiology of autism. Front Hum Neurosci 4:224, 2010.

Mills KR, Nithi KA: Corticomotor threshold to magnetic stimulation: Normal values and repeatability. Muscle Nerve 20:570-576, 1997.

Nettekoven C, Volz LJ, Kutscha M, Pool EM, Rehme AK, Eickhoff SB, Fink GR, Grefkes C: Dose-dependent effects of theta burst rTMS on cortical excitability and resting-state connectivity of the human motor system. J Neurosci 34:6849-6859, 2014.

Oberman L, Eldaief M, Fecteau S, Ifert-Miller F, Tormos JM, PascualLeone A: Abnormal modulation of corticospinal excitability in adults with Asperger's syndrome. Eur J Neurosci 36:2782-2788, 2012.

Oberman L, Ifert-Miller F, Najib U, Bashir S, Woollacott I, GonzalezHeydrich J, Picker J, Rotenberg A, Pascual-Leone A: Transcranial magnetic stimulation provides means to assess cortical plasticity and excitability in humans with fragile $\mathrm{x}$ syndrome and autism spectrum disorder. Front Synaptic Neurosci 2:26, 2010.

Oberman LM, Pascual-Leone A, Rotenberg A: Modulation of corticospinal excitability by transcranial magnetic stimulation in children and adolescents with autism spectrum disorder. Front Hum Neurosci 8:627, 2014.

Oberman LM, Rotenberg A, Pascual-Leone A: Use of transcranial magnetic stimulation in autism spectrum disorders. J Autism Dev Disord 45, 524-536, 2015.

Oldfield RC: The assessment and analysis of handedness: The edinburgh inventory. Neuropsychologia 9:97-113, 1971.

Pedapati E, Gilbert DL, Horn PS, Huddleston DA, Laue C, Shahana $\mathrm{N}, \mathrm{Wu}$ SW: Effect of $30 \mathrm{~Hz}$ theta burst transcranial magnetic stimulation on the primary motor cortex in children and adolescents. Front Hum Neurosci 9: 91, 2015.

Player MJ, Taylor JL, Alonzo A, Loo CK: Paired associative stimulation increases motor cortex excitability more effectively than theta-burst stimulation. Clin Neurophysiol 123:2220-2226, 2012.

Reid CS, Serrien DJ: Handedness and the excitability of cortical inhibitory circuits. Behav Brain Res 230:144-148, 2012.
Roid G: Stanford-Binet Intelligence Scales. Fifth ed. Itasca (Illinois), Riverside, 2003.

Rossi S, Hallett M, Rossini PM, Pascual-Leone A: Screening questionnaire before TMS: An update. Clin Neurophysiol 122:1686, 2011.

Spooren W, Lindemann L, Ghosh A, Santarelli L: Synapse dysfunction in autism: A molecular medicine approach to drug discovery in neurodevelopmental disorders. Trends Pharmacol Sci 33:669-684, 2012.

Stephenson DT, O’Neill SM, Narayan S, Tiwari A, Arnold E, Samaroo HD, Du F, Ring RH, Campbell B, Pletcher M, Vaidya VA, Morton D: Histopathologic characterization of the BTBR mouse model of autistic-like behavior reveals selective changes in neurodevelopmental proteins and adult hippocampal neurogenesis. Mol Autism 2:7, 2011.

Teitelbaum P, Teitelbaum O, Nye J, Fryman J, Maurer RG: Movement analysis in infancy may be useful for early diagnosis of autism. Proc Natl Acad Sci U S A 95:13982-13987, 1998.

Théoret H, Halligan E, Kobayashi M, Fregni F, Tager-Flusberg H, Pascual-Leone A: Impaired motor facilitation during action observation in individuals with autism spectrum disorder. Curr Biol 15:R84-R85, 2005.

Vernet M, Bashir S, Yoo WK, Oberman L, Mizrahi I, Ifert-Miller F, Beck CJ, Pascual-Leone A: Reproducibility of the effects of theta burst stimulation on motor cortical plasticity in healthy participants. Clin Neurophysiol 125:320-326, 2014.

Vernet M, Bashir S, Yoo WK, Perez JM, Najib U, Pascual-Leone A: Insights on the neural basis of motor plasticity induced by theta burst stimulation from TMS-EEG. Eur J Neurosci 37:598-606, 2013.

Walsh CA, Morrow EM, Rubenstein JL. Autism and brain development. Cell 135:396-400, 2008.

Wang J, Barstein J, Ethridge LE, Mosconi MW, Takarae Y, Sweeney JA: Resting state EEG abnormalities in autism spectrum disorders. J Neurodev Disord 5:24-24, 2013.

Wetmore DZ, Garner CC: Emerging pharmacotherapies for neurodevelopmental disorders. J Dev Behav Pediatr 31:564-581, 2010.

Won H, Lee H-R, Gee HY, Mah W, Kim J-I, Lee J, Ha S, Chung C, Jung ES, Cho YS, Park S-G, Lee J-S, Lee K, Kim D, Bae YC, Kaang BK, Lee MG, Kim E: Autistic-like social behaviour in Shank2-mutant mice improved by restoring NMDA receptor function. Nature 486:261-265, 2012.

Wu SW, Shahana N, Huddleston DA, Gilbert DL: Effects of $30 \mathrm{~Hz}$ theta burst transcranial magnetic stimulation on the primary motor cortex. J Neurosci Methods 208:161-164, 2012.

Wu SW, Shahana N, Huddleston DA, Lewis AN, Gilbert DL: Safety and tolerability of theta-burst transcranial magnetic stimulation in children. Dev Med Child Neurol 54:636-639, 2012.

Yang X-R, Kirton A, Wilkes TC, Pradhan S, Liu I, Jaworska N, Damji O, Keess J, Langevin LM, Rajapakse T: Glutamate alterations associated with transcranial magnetic stimulation in youth depression: A case series. J ECT 30:242-247, 2014.

Zafar N, Paulus W, Sommer M: Comparative assessment of best conventional with best theta burst repetitive transcranial magnetic stimulation protocols on human motor cortex excitability. Clin Neurophysiol 119:1393-1399, 2008.

Ziemann U, Reis J, Schwenkreis P, Rosanova M, Strafella A, Badawy R, Muller-Dahlhaus F: TMS and drugs revisited 2014. Clin Neurophysiol 126:1847-1868, 2015.

Address correspondence to: Ernest V. Pedapati, $M D, M S$ MLC 3014 3333 Burnett Avenue Cincinnati, $\mathrm{OH} 45229$

E-mail: ernest.pedapati@cchmc.org 